OPEN ACCESS

Edited by:

Human Mousavi Fatemi, ART Fertility Clinics,

United Arab Emirates

Reviewed by: Kazem Nouri,

University of Vienna, Austria Desislava Markova, ART Fertility Clinics $L L C$, United Arab Emirates

*Correspondence: Jun Zhai

bestzhai2005@163.com

Specialty section: This article was submitted to Reproduction,

a section of the journa Frontiers in Endocrinology

Received: 19 November 2020 Accepted: 18 January 2021 Published: 02 March 2021

Citation:

Yue M, Ma L-N, Cao Y-R and Zhai $J$ (2021) Application of a Nomogram for Predicting the Risk of Subchorionic Hematoma in Early Pregnancy With In Vitro Fertilization-Embryo Transfer/ Frozen Embryo Transfer.

Front. Endocrinol. 12:631097. doi: 10.3389/fendo.2021.631097

\section{Application of a Nomogram for Predicting the Risk of Subchorionic Hematoma in Early Pregnancy With In Vitro Fertilization-Embryo Transfer/Frozen Embryo Transfer}

\author{
Ma Yue ${ }^{1,2,3}$, Linna Ma ${ }^{1,2,3}$, Yurong Cao ${ }^{1,2,3}$ and Jun Zhai ${ }^{1,2,3 *}$ \\ ${ }^{1}$ Center for Reproductive Medicine, The First Affiliated Hospital of Zhengzhou University, Zhengzhou, China, ${ }^{2}$ Henan Key \\ Laboratory of Reproduction and Genetics, The First Affiliated Hospital of Zhengzhou University, Zhengzhou, China, ${ }^{3}$ Henan \\ Provincial Obstetrical and Gynecological Diseases (Reproductive Medicine) Clinical Research Center, The First Affiliated \\ Hospital of Zhengzhou University, Zhengzhou, China
}

Background: Subchorionic hematoma $(\mathrm{SCH})$ is common in early pregnancy achieved by in vitro fertilization-embryo transfer/frozen embryo transfer (IVF-ET/FET), and is associated with adverse obstetric outcomes. However, there are no methods known to accurately predict the occurrence of $\mathrm{SCH}$.

Objective: To establish a nomogram prediction model for predicting the risk of $\mathrm{SCH}$ in early pregnancy with IVF-ET/FET and to analyze pregnancy outcomes of patients with SCH.

Methods: Patients who underwent IVF-ET/FET treatment and were diagnosed with clinical pregnancy were enrolled in our study. A total of 256 patients with $\mathrm{SCH}$ were enrolled in the $\mathrm{SCH}$ group, and 526 patients without $\mathrm{SCH}$ in the control group. Logistic regression was used to screen risk factors for $\mathrm{SCH}$, and the nomogram was developed according to the regression coefficient of relevant variables. Discrimination, effect, calibration, and the predictive model's clinical usefulness were assessed using the C-index, the area under the receiver operating characteristic standard curve, calibration plot, and decision curve analysis. Internal validation was assessed using bootstrapping validation. The effects of $\mathrm{SCH}$ on pregnancy outcomes were analyzed.

Results: A multivariate logistic regression analysis showed that fresh embryo transfer, polycystic ovary syndrome, hydrosalpinx, and thin endometrium were risk factors affecting the occurrence of $\mathrm{SCH}$. Based on the above factors, a predictive model for the risk of $\mathrm{SCH}$ was created. The model displayed good discrimination, with a C-index of 0.783 (95\% confidence interval: 0.750-0.816), area under the receiver operating characteristic standard curve of 0.783 , and good calibration. A high C-index value of 0.765 could still be reached in the interval validation. Decision curve analysis showed that the nomogram was clinically useful when the intervention was decided at the $\mathrm{SCH}$ possibility threshold of $4 \%-87 \%$. For patients with successful deliveries, the occurrence of $\mathrm{SCH}$ did not 
influence the gestational weeks of delivery, mode of delivery, preterm birth, height, and weight of the newborn.

\begin{abstract}
Conclusion: We screened the risk factors for $\mathrm{SCH}$ in patients who underwent IVF-ET/FET treatment. Successful establishment of a nomogram can effectively predict the occurrence of $\mathrm{SCH}$. Furthermore, the incidence of miscarriage is higher in patients with $\mathrm{SCH}$.
\end{abstract}

Keywords: subchorionic hematoma, IVF-ET, FET, nomogram, decision curve analysis

\section{INTRODUCTION}

Subchorionic hematoma ( $\mathrm{SCH}$ ) in early pregnancy is a commonly observed feature on ultrasound. The mechanism causing SCH is believed to be the partial detachment of the chorionic membrane from the decidual membrane, resulting in blood accumulation between the chorionic membrane and the sacral membrane, resulting in the formation of a hematoma (1). Under pelvic ultrasound, $\mathrm{SCH}$ usually manifests as a hypoechoic or echo-free area between the chorionic membrane and the myometrium (mostly crescent-shaped). If the hematoma is large and clots have formed, petechial or linear hyperechoic areas can be detected around the gestational sac and under the chorionic membrane, whose lower edges are mostly connected to the inner cervical canal. The existence of $\mathrm{SCH}$ is possibly related to the occurrence of adverse obstetric outcomes, such as miscarriage (2). However, the induction of $\mathrm{SCH}$ remains unclear, and the occurrence of $\mathrm{SCH}$ is reportedly related to thrombosis (3).

Further studies have shown that $\mathrm{SCH}$ is associated with mesenchymal dysplasia and vaginal dysbacteriosis $(4,5)$. The reported incidence of $\mathrm{SCH}$ in pregnancy is $4 \%-48 \%$, and clinical symptoms appear at 8-33 weeks of pregnancy (6). A study found that the incidence of $\mathrm{SCH}$ is higher in pregnancies achieved by in vitro fertilization and embryo transfer than in natural pregnancies (7).

Risk factors affecting $\mathrm{SCH}$ are unclear. There is a lack of intuitive and effective methods to assess the risk of $\mathrm{SCH}$. The aim of the present study was to establish a nomogram for predicting the risk of occurrence of $\mathrm{SCH}$ in early pregnancies after in vitro fertilization-embryo transfer/frozen embryo transfer (IVF-ET/ FET). The second objective was to evaluate the pregnancy outcomes of women with SCH.

\section{MATERIALS AND METHODS}

\section{Data Extraction}

We retrieved data through the clinical reproductive medicine management system at the reproductive center of the First Affiliated Hospital of Zhengzhou University. During the study period from September 1, 2017, to October 31, 2017, 782 pregnancies achieved by IVF-ET/FET were included in the study.

Abbreviations: IVF-ET/FET, in vitro fertilization-embryo transfer/frozen embryo transfer; PCOS, Polycystic ovary syndrome; SCH, Subchorionic hematoma.
All patients underwent ultrasound examinations on days 35 and 45 after embryo transfer. Clinical pregnancy was defined as the presence of a gestational sac in the uterus by ultrasound. Under ultrasound, the $\mathrm{SCH}$ appeared as a crescent-shaped, sonolucent fluid collection between the chorion or placenta and the myometrium (8). Hydrosalpinx was defined as a distally occluded tube that was pathologically dilated or became pathologically dilated when patency was tested by ultrasound, hysterosalpingography, or laparoscopy (9). Preterm delivery was defined as delivery occurring at less than 37 weeks and more than 28 weeks of gestation, or birth weight $\geq 1,000 \mathrm{~g}$. Miscarriage was defined as delivery occurring at less than 28 weeks of gestation and birth weight $\leq 1,000 \mathrm{~g}$. A total of 256 patients with SCH were enrolled in the SCH group and 526 patients without $\mathrm{SCH}$ in the control group. Gonadotropin releasing hormone (GnRH) agonist down-regulation long protocols were used in the fresh cycle. The long-acting GnRH-a (33.75 mg, Triptorelin, Beaufour Ipsen, France) was used for pituitary downregulation on the $2^{\text {nd }}$ to $3^{\text {rd }}$ days of menstruation, and pelvic ultrasound and endocrine examination were re-examined after 28-35 days. If the endocrine indexes reached the standard for pituitary downregulation, 75-300 U of recombinant human FSH (Gonal-F, Merck Serono, Switzerland) were administered for controlled ovulation hyperstimulation. When the diameters of at least two dominant follicles were $>18 \mathrm{~mm}$ or those of $2 / 3$ follicles were $>16 \mathrm{~mm}, 4,000-10,000 \mathrm{U}$ of HCG was administered. At $36-38 \mathrm{~h}$ after injection, a transvaginal ultrasoundguided oocyte-pickup puncture procedure was performed. In the frozen-thawed embryo transfer, the natural cycle was used for patients with regular menstrual cycles. The hormone replacement cycle was used for patients with an irregular menstrual cycle or who were detected to have follicle non-growth or poor growth. Embryo transfer was performed on day 3 or day 5 after embryo formation. Luteal phase support was provided via vaginal administration of progesterone gel (Crinone, Merck Serono, Switzerland) once per day and oral dydrogesterone tablets (10 mg, Duphaston, Abbott, Netherlands) twice daily. Patients with recurrent spontaneous abortions, abnormal chromosomes, immune function abnormality, and a thrombosis history were excluded.

\section{Statistical Analysis}

Statistical analysis was performed using SPSS 25.0 and R 4.0.1 software. Categorical variables were evaluated using a chi-square test, and continuous variables were assessed using the independent-samples Student's t-test. We used logistic regression to screen the risk factors of $\mathrm{SCH}$ and the developed nomogram according to the regression coefficient of the relevant variables. The area under the receiver operating characteristic standard curve (AUC) was calculated to evaluate the prediction 
accuracy of the SCH nomogram model (10). Harrell's C-index was evaluated to quantify the discrimination performance of the SCH nomogram (11), and bootstrapping validation was conducted to calculate a relatively corrected C-index (12). A calibration curve was used to measure the calibration of the SCH nomogram (13). Decision curve analysis was conducted to evaluate the clinical usefulness of the SCH nomogram by assessing the net benefits at different threshold probabilities (14). The types of investigated pregnancy outcomes included miscarriage rates (both early and late miscarriages), gestational weeks, neonatal weight, neonatal height, preterm birth rate, and mode of delivery. A $P$ value $<0.05$ was considered statistically significant.

\section{RESULTS}

\section{Patients' Characteristics}

The baseline characteristics were similar between the two groups (Table 1). The prevalence of SCH in the study was $32.7 \%$ (256/ 782). Among the patients included in the study, 390 fresh and 392 frozen-thawed embryo transfers met the inclusion criteria. The frequency of SCH was higher with fresh embryo transfers (36.41\% vs. $29.08 \%, p=0.029$ ). The endometrium thickness before transfer (the day of HCG administration) was thinner in the SCH group $(11.44 \pm 2.86$ vs. $11.95 \pm 2.0, \mathrm{p}=0.013)$. Of the women who had fresh embryo transfers, the number of ova obtained was higher in the SCH group $(13.32 \pm 6.56$ vs. $11.99 \pm$ $5.46, \mathrm{p}=0.032$ ). According to the infertility diagnosis, pregnancies in the study were divided into uterine malformation, uterine leiomyoma, intrauterine adhesion, hydrosalpinx (after the laparoscopic proximal tubal ligation), endometriosis, polycystic ovary syndrome (PCOS), male factor, and oviduct obstruction groups. The incidence of SCH was higher in the hydrosalpinx ( $40.24 \%$ vs. $30.74 \%, \mathrm{p}=0.021)$ and PCOS (38.86\% vs. $30.47 \%$, $\mathrm{p}=0.026)$ groups than in the other groups (Table 2 ).

\section{Logistic Regression Analysis and Development of a Nomogram Prediction Model}

Logistic regression analysis demonstrated that fresh embryo transfer, hydrosalpinx, PCOS, and thin endometrium were independent risk factors for $\mathrm{SCH}$ (Table 3). The prediction model was developed based on these factors and presented as a nomogram (Figure 1).

\section{Apparent Performance and Clinical Use of the SCH Nomogram}

The C-index for the nomogram was 0.783 (95\% CI: 0.750-0.816) and was verified to be 0.765 through bootstrapping validation, which indicated that the model had great discrimination. The AUC of the nomogram was 0.783 , suggesting a good prediction capability (Figure 2). The calibration curve of the nomogram for the prediction of SCH risk was proven to be in good agreement (Figure 3). The decision curve showed that if the threshold probability of a patient and a doctor is $>4 \%$ and $<87 \%$, respectively, the use of the nomogram to predict $\mathrm{SCH}$ risk is more beneficial than the intervention-all-patient scheme or the intervention-none scheme (Figure 4).

TABLE 1 | Baseline characteristics.

\begin{tabular}{|c|c|c|c|c|}
\hline Factor & SCH group $(n=256)$ & Control group $(n=526)$ & t value $/ \chi^{2}$ value & $P$ value \\
\hline Age $^{\mathrm{a}}$ (years) & $30.29 \pm 4.80$ & $30.51 \pm 4.74$ & 0.613 & 0.540 \\
\hline $\mathrm{BMI}^{\mathrm{a}}\left(\mathrm{kg} / \mathrm{m}^{2}\right)$ & $28.23 \pm 5.85$ & $28.47 \pm 5.56$ & 0.571 & 0.568 \\
\hline $\mathrm{AMH}^{\mathrm{a}}(\mathrm{ng} / \mathrm{ml})$ & $3.99 \pm 2.99$ & $3.92 \pm 3.24$ & 0.869 & 0.771 \\
\hline $\mathrm{FSH}^{\mathrm{a}}(\mathrm{IU} / \mathrm{L})$ & $6.88 \pm 2.40$ & $6.71 \pm 2.20$ & 0.937 & 0.349 \\
\hline \multicolumn{5}{|l|}{ Infertility type ${ }^{b}(n, \%)$} \\
\hline Primary & 132(33.25) & 265(66.75) & 0.096 & 0.756 \\
\hline \multicolumn{5}{|l|}{ Secondary } \\
\hline \multicolumn{5}{|l|}{ Type of embryo transfer ${ }^{b}(n, \%)$} \\
\hline $\begin{array}{l}\text { Fresh embryo transfer } \\
\text { Frozen-thawed embryo transfer } \\
\text { Number of gestational sacs }{ }^{b}(n,\end{array}$ & $\begin{array}{l}142(36.41) \\
114(29.08)\end{array}$ & $\begin{array}{l}248(63.59) \\
278(70.92)\end{array}$ & 4.768 & 0.029 \\
\hline $\begin{array}{l}\text { Single gestational sac } \\
\text { Multiple gestational sac }\end{array}$ & $\begin{array}{l}173(34.60) \\
83(28.26)\end{array}$ & $\begin{array}{l}352(65.40) \\
174(71.74)\end{array}$ & 0.034 & 0.854 \\
\hline Duration of $\mathrm{Gn}^{\mathrm{a}}$ (days) & $13.83 \pm 5.74$ & $13.87 \pm 5.73$ & 0.312 & 0.967 \\
\hline Dose of $\mathrm{Gn}^{\mathrm{a}}(\mathrm{IU})$ & $2264.06 \pm 1036.04$ & $2431.35 \pm 931.99$ & 0.826 & 0.411 \\
\hline The number of ova obtained ${ }^{\mathrm{a}}$ & $13.32 \pm 6.56$ & $11.99 \pm 5.46$ & 2.158 & 0.032 \\
\hline \multicolumn{5}{|c|}{ Type of Frozen-thawed transfer ${ }^{\mathrm{b}}$ (n, \%) } \\
\hline Natural cycle & $45(27.43)$ & $119(72.57)$ & 0.369 & 0.544 \\
\hline Hormone replacement cycle & 69(30.26) & 159(69.74) & & \\
\hline Endometrium thickness ${ }^{a}$ (mm) & $11.44 \pm 2.86$ & $11.95 \pm 2.60$ & 2.494 & 0.013 \\
\hline
\end{tabular}

a Mean $\pm S D$, comparison with the use of independent-samples $T$ test.

${ }^{b}$ Using Chi-square test.

SCH, subchorionic hematoma; BMI, body mass index; AMH, anti-müllerian hormone; FSH, follicle stimulating hormone; Gn, Gonadotropin. 
TABLE 2 | Infertility diagnosis.

\begin{tabular}{|c|c|c|c|c|}
\hline Diagnosis & $\mathrm{SCH}$ group (n, \%) & Control group (n, \%) & $\chi^{2}$ value & $P$ value \\
\hline Yes & $25(9.77)$ & $58(11.03)$ & 0.289 & 0.591 \\
\hline \multicolumn{5}{|c|}{ Uterine leiomyoma } \\
\hline Yes & $54(21.09)$ & 118(22.43) & 0.180 & 0.671 \\
\hline No & 202(78.91) & $408(77.57)$ & & \\
\hline \multicolumn{5}{|c|}{ Intrauterine adhesion } \\
\hline \multicolumn{5}{|c|}{ Hydrosalpin $x^{a}$} \\
\hline Yes & 66(25.78) & 98(18.63) & 5.312 & 0.021 \\
\hline No & $190(74.22)$ & $428(81.37)$ & & \\
\hline \multicolumn{5}{|c|}{ Endometriosis $^{a}$} \\
\hline Yes & $65(25.40)$ & 152(28.90) & 1.056 & 0.304 \\
\hline No & $191(74.60)$ & $374(71.10)$ & & \\
\hline Yes & 63(24.61) & 133(16.92) & 0.042 & 0.838 \\
\hline No & 193(75.39) & 393(83.08) & & \\
\hline \multicolumn{5}{|c|}{ Oviduct obstruction ${ }^{a}$} \\
\hline Yes & $68(26.56)$ & 137(26.05) & 0.024 & 0.877 \\
\hline No & $188(73.44)$ & 389(73.95) & & \\
\hline
\end{tabular}

aUsing Chi-square test.

$\mathrm{SCH}$, subchorionic hematoma; PCOS, polycystic ovary syndrome.

TABLE 3 | Multivariate logistic regression analysis.

\begin{tabular}{|c|c|c|c|c|}
\hline Factor & Regression coefficients $(\beta)$ & Significance (P) & OR & OR $95 \%$ confidence interval \\
\hline Hydrosalpinx & 0.369 & 0.044 & 1.45 & $1.01-2.07$ \\
\hline PCOS & 0.336 & 0.048 & 1.40 & $1.00-1.95$ \\
\hline Cycle type & 0.355 & 0.023 & 1.43 & $1.05-1.94$ \\
\hline Endometrial thickness & 0.075 & 0.011 & 0.93 & $0.88-0.98$ \\
\hline
\end{tabular}

PCOS, polycystic ovary syndrome; OR, odds ratio.

\section{Pregnancy Outcomes}

The miscarriage rate was significantly higher in the $\mathrm{SCH}$ group than in the control group $(24.61 \%$ vs. $15.40 \%, \mathrm{P}=0.002)$. However, the delivery gestational age, neonatal height, neonatal weight, preterm birth rate, and delivery mode were not significantly different between the two groups (the miscarriage pregnancies were excluded, Table 4). After excluding pregnancies with uterine malformation, PCOS, endometriosis, and hydrosalpinx that may influence the miscarriage rate, 219 patients with male factors and oviduct obstruction were retained. Among them, 63 pregnancies were enrolled in the SCH group and 156 were included in the control group. The miscarriage rate was still significantly higher in the $\mathrm{SCH} \operatorname{group}(25.40 \%$ vs. $13.46 \%, \mathrm{P}=0.035)$.

\section{DISCUSSION}

Subchorionic hematoma is common in pregnancies achieved by IVF-ET/FET; however, the mechanism causing SCH is unclear. Risk factors affecting the incidence of $\mathrm{SCH}$ and pregnancy outcomes of patients with $\mathrm{SCH}$ in assisted reproductive technology have remained unclear. Our study showed that the incidence of $\mathrm{SCH}$ was significantly higher in pregnancies achieved by fresh embryo transfer than in those achieved by frozen-thawed embryo transfers. PCOS, hydrosalpinx, and thin endometrium were independent risk factors affecting the occurrence of $\mathrm{SCH}$, and the miscarriage rate increased significantly in patients with $\mathrm{SCH}$.

Zhou et al. reported that the incidence of SCH was higher in patients receiving fresh embryo transfer (15). In contrast, Asato et al. showed that the incidence was higher in pregnancies achieved by frozen-thawed embryo transfer (7). However, most researchers believe that the incidence of obstetric complications is lower with frozen-thawed embryo transfer, and the obstetric outcomes are also better for frozen-thawed embryo transfers (16, 17). Some studies have suggested that the relatively natural womb environment during frozen-thawed embryo transfer is more conducive to embryo implantation and placenta formation $(18,19)$. In contrast, ovarian hyperstimulation in fresh embryo transfer affects the generation and implantation of endometrial blood vessels (20). A study showed that the incidence of vaginal 

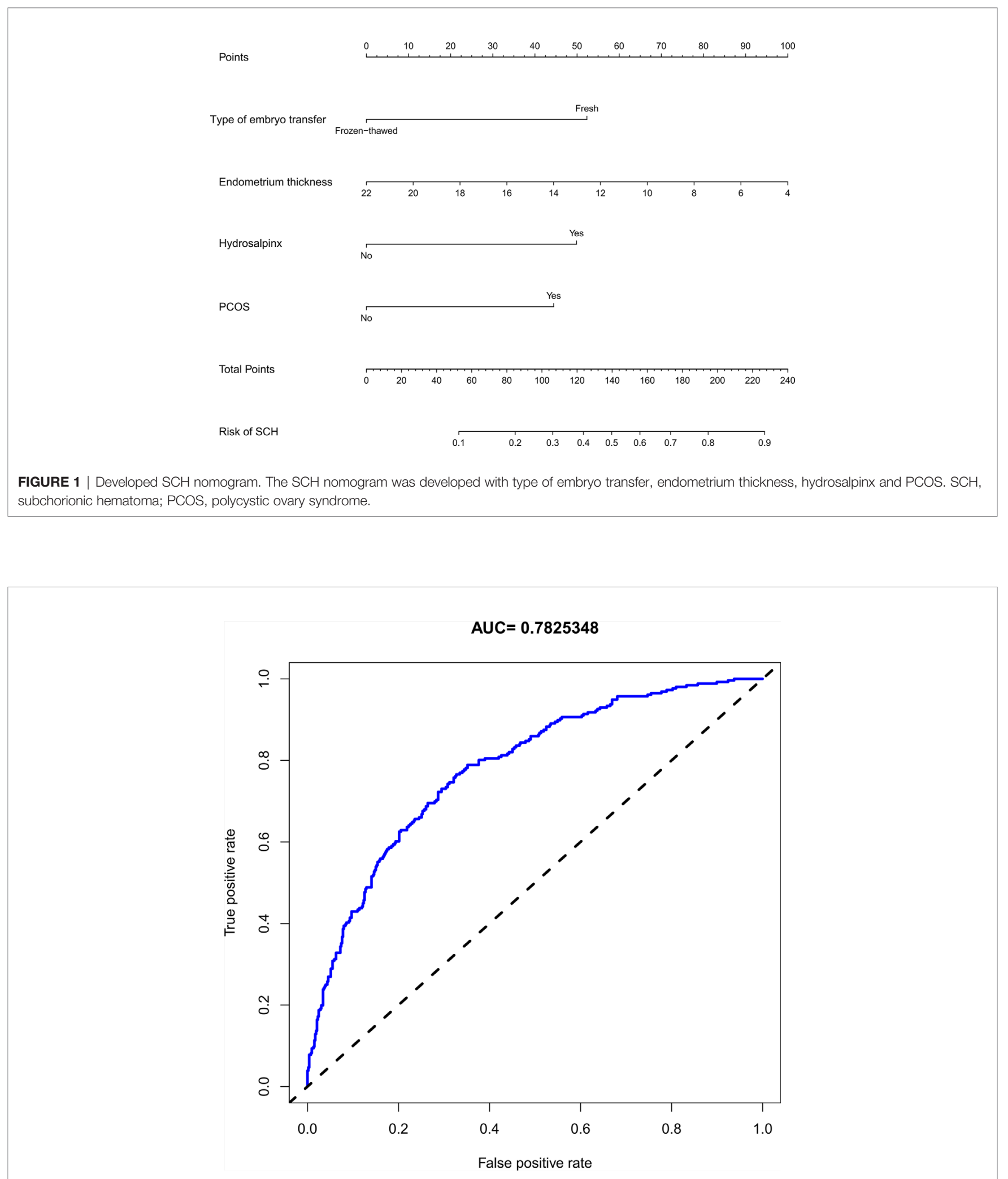

FIGURE 2 | The receiver operating characteristic of the subchorionic hematoma (SCH) nomogram. AUC, the area under the receiver operating characteristic standard curve. 


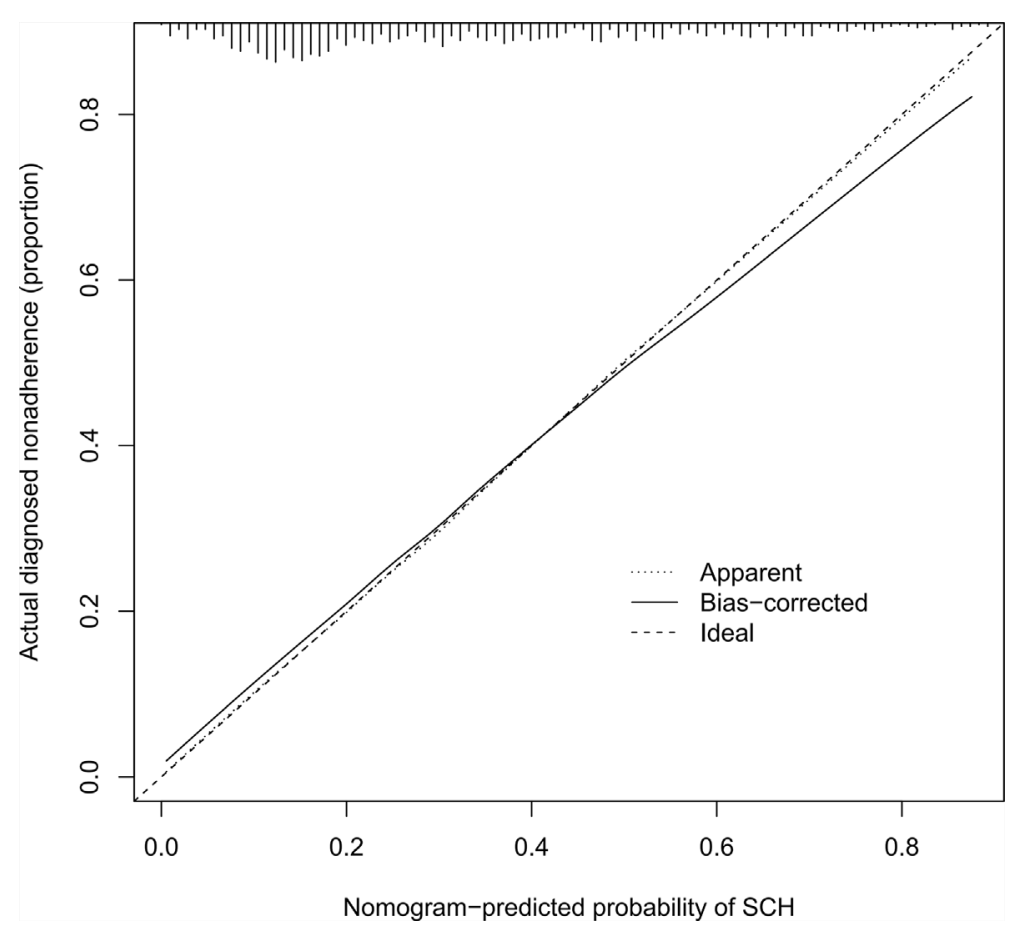

FIGURE 3 | Calibration curves of the SCH nomogram. The x-axis represents the predicted risk for SCH. The y-axis represents the actual diagnosed SCH. The diagonal dotted line represents a perfect prediction by an ideal model. The solid line represents the performance of the nomogram, of which a closer fit to the diagonal dotted line represents a better prediction. SCH, subchorionic hematoma.

bleeding in pregnancies achieved by fresh embryo transfer cycle increases with the number of ova obtained (21). This probably resulted from the higher estrogen levels in patients with more ova obtained, disturbing the function of endometrial blood vessels. Our study also showed that the incidence of SCH was higher in pregnancies achieved by fresh embryo transfer and increased with the number of ova obtained. The estradiol level was also higher in the $\mathrm{SCH}$ group, but the difference was not significant $(\mathrm{p}=0.556)$. As for the frozen-thawed embryo transfer cycle, the incidence of SCH was higher in the hormone replacement cycle, but the difference was not significant ( $30.26 \%$ vs. $27.43 \%, p=0.544$ ). This could be due to the small sample sizes. Reich et al. also confirmed that the incidence was higher in the hormone replacement cycle (22). In addition, endometrium thickness on the day of HCG administration, which is correlated with endometrial receptivity, reflects the function of the endometrium and is associated with endometrial receptivity $(23,24)$. Our study showed that endometrium thickness was lower in the $\mathrm{SCH}$ group $(\mathrm{p}=0.013)$.

In the present study, all patients in the hydrosalpinx group were treated with tubal ligation in order to avoid the toxic effect and mechanical erosion of the hydrosalpinx fluid on the endometrium. Salpingectomy was not performed considering its potential impact on ovarian function. The hydrosalpinx could not return to the uterine cavity, resulting in toxic effects and mechanical erosion. Some pathogenic factors, such as cytokines, prostaglandins, leukocyte chemokines, and other inflammatory factors, are induced by the hydrops and act on adjacent organs through lymph and blood circulation, thus disrupting the function of the endometrium (25). The expression levels of integrin $\alpha_{\gamma} \beta_{3}$, leukemia inhibitory factor, and homeobox gene A10 (HOXA10) are significantly decreased in patients with hydrosalpinx $(26,27)$. Therefore, hydrosalpinx might disturb endometrial receptivity in various ways, leading to $\mathrm{SCH}$. Likewise, PCOS might induce SCH by influencing endometrial receptivity (28). Some studies have suggested that the expression of integrin $\alpha_{\gamma} \beta_{3}$, interleukin 6 family cytokine (LIF), and HOXA10 genes decrease in patients with PCOS (29). Insulin resistance and hyperinsulinemia play a key role in PCOS physiology and pathology. Lathi et al. found that high insulin levels can reduce the expression of insulin-like growth factorbinding protein-1 (IGFBP-1) (30). A study showed that with high insulin levels, the expression of integrin and osteopontin also shows a decreasing trend (31).

Among studies on assisted reproduction in humans, few have evaluated the effects of SCH on pregnancy outcomes in women who underwent IVF-ET/FET $(15,32)$. A study reported that the presence of SCH in patients who underwent IVF-ET/FET had no influence on the incidence of spontaneous miscarriage or live birth rate (15). As for natural pregnancies, however, most studies have suggested a significant correlation between $\mathrm{SCH}$ and adverse obstetric outcomes $(2,33)$. Moreover, the occurrence of $\mathrm{SCH}$ could increase the risk of spontaneous miscarriage, premature delivery, placental abruption, and gestational 


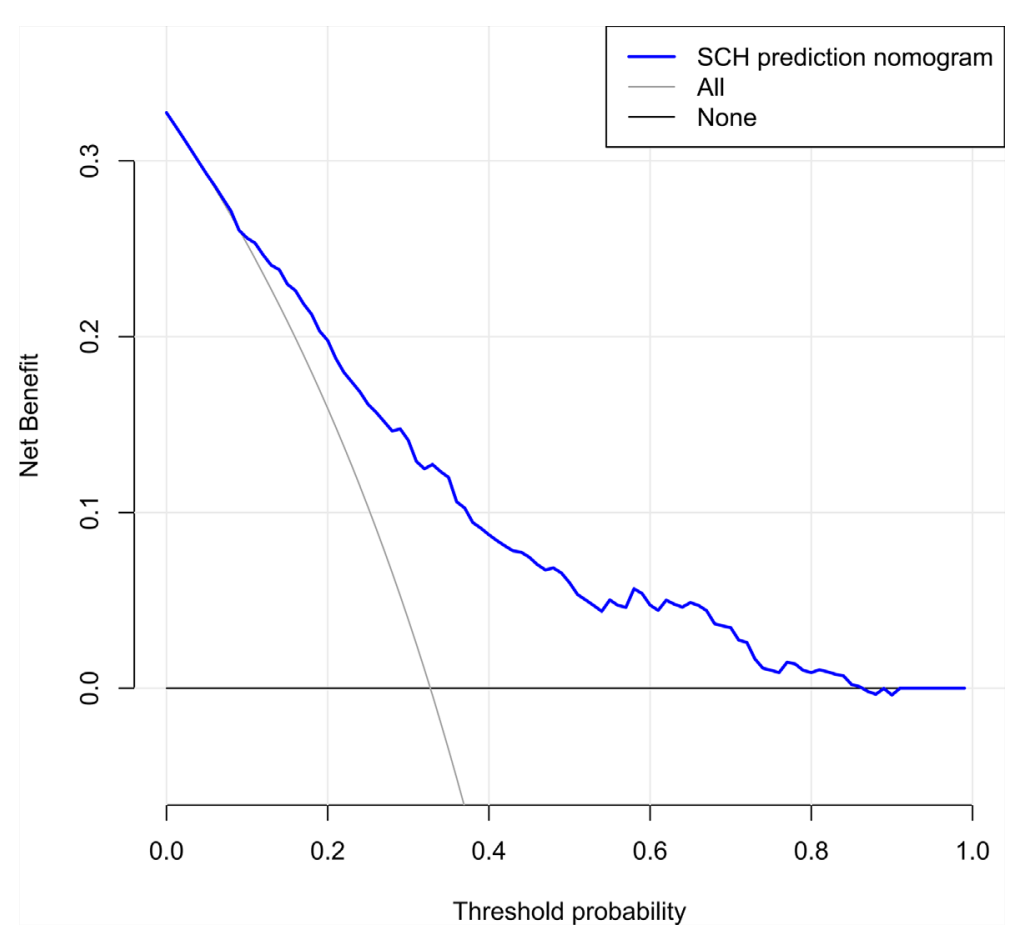

FIGURE 4 | Decision curve analysis for the SCH nomogram. The y-axis measures the net benefit. The dotted line represents the SCH risk nomogram. The thin light solid line represents the assumption that all patients are diagnosed as $\mathrm{SCH}$. The thin thick solid line represents the assumption that no patients are diagnosed as $\mathrm{SCH}$. The decision curve showed that if the threshold probability of a patient and a doctor is > 4 and $<87 \%$, respectively, using this nomogram in the current study to predict risk for $\mathrm{SCH}$ adds more benefit than the intervention-all-patients scheme or the intervention-none scheme. SCH, subchorionic hematoma.

TABLE 4 | Pregnancy outcomes.

\begin{tabular}{|c|c|c|c|c|}
\hline Outcomes & SCH group $(n=256)$ & Control group $(n=526)$ & $\mathrm{t}$ value $/ \chi^{2}$ value & $P$ value \\
\hline Miscarriage rates ${ }^{\mathrm{b}}[\%(\mathrm{n} / \mathrm{N})]$ & 24.61(63/256) & $15.40(81 / 526)$ & 9.723 & 0.002 \\
\hline Gestational weeks ${ }^{b}$ (week) & $37.72 \pm 2.17$ & $37.94 \pm 1.90$ & 1.299 & 0.194 \\
\hline Neonatal weight ${ }^{\mathrm{b}}(\mathrm{g})$ & $3264.97 \pm 474.60$ & $3203.26 \pm 494.59$ & 1.476 & 0.140 \\
\hline Neonatal height ${ }^{\mathrm{b}}(\mathrm{cm})$ & $49.59 \pm 1.99$ & $49.88 \pm 1.98$ & 1.668 & 0.096 \\
\hline Preterm birth rate ${ }^{\mathrm{b}}[\%(\mathrm{n} / \mathrm{N})]$ & 16.58(32/193) & $14.83(66 / 445)$ & 0.317 & 0.574 \\
\hline \multicolumn{5}{|l|}{ Mode of delivery $(n, \%)$} \\
\hline Cesarean section & $149(77.20)$ & 355(79.78) & 0.537 & 0.464 \\
\hline Natural delivery & $44(22.80)$ & $90(20.22)$ & & \\
\hline
\end{tabular}

aMean $\pm S D$, comparison with the use of independent-samples $T$ test.

${ }^{b}$ Using Chi-squared test.

$\mathrm{SCH}$, subchorionic hematoma.

hypertension (34-37). Based on our findings, $\mathrm{SCH}$ was also found to be an independent risk factor for miscarriage.

To the best of our knowledge, this was the first study that applied the nomogram prediction model in predicting the risk of SCH in early pregnancy with IVF-ET/FET. The nomogram had good predictive efficacy and guidance significance for clinical decisions. With the nomogram, we could intuitively assess the risk of SCH and administer individualized treatments for highrisk patients. For example, a patient with hydrosalpinx would have a score of 50 based on our nomogram, corresponding to a $10 \%$ risk of $\mathrm{SCH}$. In a patient with a combination of two risk factors (PCOS and FET), the total score is 97, which corresponds to a $26 \%$ risk of SCH. Regarding patients with a high risk of SCH during the first trimester, monitoring should be enhanced to detect $\mathrm{SCH}$ as soon as possible and take timely measures to reduce the occurrence of miscarriage.

\section{DATA AVAILABILITY STATEMENT}

The raw data supporting the conclusions of this article will be made available by the authors, without undue reservation. 


\section{ETHICS STATEMENT}

Parameters such as medical history identifiers were recoded prior to extraction to maintain anonymized records, with no access to identifying information, in accordance with the principles of Good Clinical Practice and the Declaration of Helsinki. As such, the Ethics Committee for scientific research and clinical trials of the First Affiliated Hospital of Zhengzhou University approved the study.

\section{AUTHOR CONTRIBUTIONS}

MY, L-NM, and Y-RC performed the data collection, statistical analyses, and manuscript preparation. MY and JZ participated in the study design and manuscript preparation. All authors contributed to the article and approved the submitted version.

\section{REFERENCES}

1. Maso G, D'Ottavio G, Seta FD, Sartore A, Mandruzzato G. First-Trimester Intrauterine Hematoma and Outcome of Pregnancy. Obstetrics Gynecol (2005) 105(2):339-44. doi: 10.1097/01.AOG.0000152000.71369.bd

2. Ükür YE, Gksu G, Kse O, Amaz G, Zmen B, Atabekolu CS, et al. The effects of subchorionic hematoma on pregnancy outcome in patients with threatened abortion. J Turkish German Gynecol Assoc (2014) 15(4):239. doi: 10.5152/ jtgga.2014.14170

3. Baxi LV, Pearlstone MM. Subchorionic hematomas and the presence of autoantibodies. Am J Obstet Gynecol (1991) 165(5 Pt 1):1423-4. doi: 10.1016/ 0002-9378(91)90383-3

4. Chen CP, Hsu CY, Su YN, Wang TY, Chern SR, Su JW, et al. Placental mesenchymal dysplasia associated with antepartum hemorrhage, subchorionic hematoma, and intrauterine growth restriction. Taiwanese J Obstetrics Gynecol (2013) 52(1):154-6. doi: 10.1016/j.tjog.2013.01.023

5. Yamada T, Atsuki Y, Wakasaya A, Kobayashi M, Hirano Y, Ohwada M. Characteristics of patients with subchorionic hematomas in the second trimester. J Obstetrics Gynaecol Res (2012) 38(1):180-4. doi: 10.1111/j.14470756.2011.01665.x

6. Pearlstone M, Baxi L. Subchorionic hematoma: a review. Obstetr Gynecol Survey (1993) 48(2):65-8. doi: 10.1097/00006254-199302000-00001

7. Asato K, Mekaru K, Heshiki C, Sugiyama H, Kinjyo T, Masamoto H, et al. Subchorionic hematoma occurs more frequently in in vitro fertilization pregnancy. Eur J Obstetrics Gynecol Reprod Biol (2014) 181:41-4. doi: 10.1016/j.ejogrb.2014.07.014

8. Jemma J, Jon H, Johns J, Hyett J, Jauniaux E. Obstetric outcome after threatened miscarriage with and without a hematoma on ultrasound. Obstetrics Gynecol (2003) 102(3):483-7. doi: 10.1016/S0029-7844(03)00580-5

9. Strandell A, Lindhard A, Waldenström U, Thorburn J. Hydrosalpinx and IVF outcome: cumulative results after salpingectomy in a randomized controlled trial. Hum Reproduct (2001) 16(11):2403-10. doi: 10.1093/humrep/ 16.11.2403

10. Tolcher CM, Holbert MR, Weaver AL, McGree ME, Olson JE, El-Nashar SA, et al. Predicting Cesarean Delivery After Induction of Labor Among Nulliparous Women at Term. Obstetrics Gynecol (2015) 126(5):1059. doi: 10.1097/AOG.0000000000001083

11. Marcel W. Prognostic models with competing risks: methods and application to coronary risk prediction. Epidemiol (Cambridge Mass) (2009) 4(20):55561. doi: 10.1097/EDE.0b013e3181a39056

12. Davison AC, Hinkley DV. Bootstrap Methods and Their Application. Technometrics (1997) 94(445):216-7. doi: 10.1017/CBO9780511802843

13. Kramer AA, Zimmerman JE. Assessing the calibration of mortality benchmarks in critical care: The Hosmer-Lemeshow test revisited. Crit Care Med (2007) 35(9):2052-6. doi: 10.1097/01.CCM.0000275267.64078.B0

14. Vickers AJ, Cronin AM, Elkin EB, Gonen M. Extensions to decision curve analysis, a novel method for evaluating diagnostic tests, prediction models and

\section{FUNDING}

Funding for the work undertaken in this study was provided by a Project Grant from the National Natural Science Foundation of China (grant no. 82071649). The funders had no role in the study design, data collection and analysis, nor decision to submit the article for publication.

\section{ACKNOWLEDGMENTS}

The authors thank the Center for Reproductive Medicine of the First Affiliated Hospital of Zhengzhou University for research support. All staff and research participants at the Center for Reproductive Medicine are acknowledged with thanks.

molecular markers. BMC Med Inf Decision Making (2008) 8(1):1-17. doi: 10.1186/1472-6947-8-53

15. Zhou J, Min W, Wang B, Hou X, Wang J, Chen H, et al. The effect of first trimester subchorionic hematoma on pregnancy outcomes in patients underwent IVF/ICSI treatment. J Maternal-Fetal Med (2016) 30(4):406-10. doi: 10.1080/14767058.2016.1174682

16. Maheshwari A, Pandey S, Shetty A, Hamilton M, Bhattacharya S. Obstetric and perinatal outcomes in singleton pregnancies resulting from the transfer of frozen thawed versus fresh embryos generated through in vitro fertilization treatment: a systematic review and meta-analysis. Fertil Steril (2012) 98 (2):368. doi: 10.1016/j.fertnstert.2012.05.019

17. Antonina $\mathrm{S}$. Obstetric outcome in singletons after in vitro fertilization with cryopreserved/thawed embryos. Hum Reprod (Oxford England) (2012) 5 (27):1343-50. doi: 10.1093/humrep/des036

18. Roque M, Lattes K, Serra S, Solà I, Geber S, Carreras R, et al. Fresh embryo transfer versus frozen embryo transfer in in vitro fertilization cycles: a systematic review and meta-analysis. Fertil Steril (2013) 99(1):156-62. doi: 10.1016/j.fertnstert.2012.09.003

19. Simón C, Velasco JJG, Valbuena D, Peinado JA, Pellicer A. Increasing uterine receptivity by decreasing estradiol levels during the preimplantation period in high responders with the use of a follicle-stimulating hormone step-down regimen. Fertil Steril (1998) 70(2):234. doi: 10.1016/S0015-0282(98)00140-X

20. Kalra SK, Ratcliffe SJ, Milman L, Gracia CR, Coutifaris C, Barnhart KT. Perinatal morbidity after in vitro fertilization is lower with frozen embryo transfer. Fertil Steril (2011) 95(2):548-53. doi: 10.1016/j.fertnstert.2010.05.049

21. Healy DL, Breheny S, Halliday J, Jaques A, Rushford D, Garrett C, et al. Prevalence and risk factors for obstetric haemorrhage in 6730 singleton births after assisted reproductive technology in Victoria Australia. Hum Reprod (2009) 25(1):265-74. doi: 10.1093/humrep/dep376

22. Reich J, Blakemore JK, Grifo JA. Comparison of subchorionic hematoma in medicated or natural single euploid frozen embryo transfer cycles. Fertil Steril (2020) 114(3):595-600. doi: 10.1016/j.fertnstert.2020.04.040

23. Geyter CD, Schmitter M, Geyter MD, Nieschlag E, Schneider HPG. Prospective evaluation of the ultrasound appearance of the endometrium in a cohort of 1,186 infertile women. Fertil Steril (2000) 73(1):106-13. doi: 10.1016/S0015-0282(99)00484-7

24. Dietterich C, Check JH, Choe JK, Nazari A, Lurie D. Increased endometrial thickness on the day of human chorionic gonadotropin injection does not adversely affect pregnancy or implantation rates following in vitro fertilization-embryo transfer. Fertil Steril (2002) 77(4):781-6. doi: 10.1016/S0015-0282(01)03276-9

25. Meyer WR, Castelbaum AJ, Somkuti S, Sagoskin AW, Doyle M, Harris JE, et al. Hydrosalpinges adversely affect markers of endometrial receptivity. Hum Reprod (1997) 12(7):1393-8. doi: 10.1093/humrep/12.7.1393

26. Lu L, Xu BF, Chen QJ, Sun XX. Effects of hydrosalpinx on pinopodes, leukaemia inhibitory factor, integrin $\beta 3$ and MUC1 expression in the periimplantation endometrium. Eur J Obstetrics Gynecol Reprod Biol (2010) 151 (2):171-5. doi: 10.1016/j.ejogrb.2010.04.024 
27. Blake DA, Proctor M, Johnson N, Olive D. Cleavage stage versus blastocyst stage embryo transfer in assisted conception. Cochrane Database Systematic Rev (2005) 7(4):CD002118. doi: 10.1002/14651858.CD002118.pub2

28. Lopes IMRS, Maganhin CC, Oliveira-Filho RM, Simões RS, Simões MJ, Iwata MC, et al. Histomorphometric Analysis and Markers of Endometrial Receptivity Embryonic Implantation in Women With Polycystic Ovary Syndrome During the Treatment With Progesterone. Reprod Sci (2014) 21 (7):930-8. doi: 10.1177/1933719113519169

29. Lathi RB, Hess AP, Tulac S, Nayak NR, Conti M, Giudice LC. Dose-dependent insulin regulation of insulin-like growth factor binding protein-1 in human endometrial stromal cells is mediated by distinct signaling pathways. J Clin Endocrinol Metab (2005) 90(3):1599-606. doi: 10.1210/jc.2004-1676

30. Cakmak H, Taylor HS. Implantation failure: molecular mechanisms and clinical treatment. Hum Reprod Update (2011) 17(2):242-53. doi: 10.1093/ humupd/dmq037

31. Wu M, Yin Y, Zhao M, Hu L, Chen Q. The low expression of leukemia inhibitory factor in endometrium: possible relevant to unexplained infertility with multiple implantation failures. Cytokine (2013) 62(2):334-9. doi: 10.1016/j.cyto.2013.03.002

32. Reich J, Blakemore JK, Grifo JA. Comparison of subchorionic hematoma in medicated or natural single euploid frozen embryo transfer cycles. Fertil Steril (2020) 114(3). doi: 10.1016/j.fertnstert.2020.04.040

33. Palatnik A, Grobman WA. The relationship between first-trimester subchorionic hematoma, cervical length, and preterm birth. Am J Obstetrics Gynecol (2015) 403:e1-.e4. doi: 10.1016/j.ajog.2015.05.019
34. Jauniaux E, Van Oppenraaij RH, Burton GJ. Obstetric outcome after early placental complications. Curr Opin Obstet Gynecol (2010) 22(6):452-7. doi: 10.1097/GCO.0b013e3283404e44

35. Tuuli MG, Norman SM, Odibo AO, Macones GA, Cahill AG. Perinatal outcomes in women with subchorionic hematoma: a systematic review and meta-analysis. Obstetrics Gynecol (2011) 117(5):1205-12. doi: 10.1097/ AOG.0b013e31821568de

36. Janowiczgrelewska A, Sieroszewski P. [Prognostic significance of subchorionic hematoma for the course of pregnancy]. Ginekol Polska (2013) 84(11):944-9. doi: $10.17772 / \mathrm{gp} / 1664$

37. Nagy S, Bush M, Stone J, Lapinski RH, Gardó S. Clinical significance of subchorionic and retroplacental hematomas detected in the first trimester of pregnancy. Obstetrics Gynecol (2003) 102(1):94-100. doi: 10.1097/00006250200307000-00019

Conflict of Interest: The authors declare that the research was conducted in the absence of any commercial or financial relationships that could be construed as a potential conflict of interest.

Copyright (c) $2021 \mathrm{Yue}, \mathrm{Ma}$, Cao and Zhai. This is an open-access article distributed under the terms of the Creative Commons Attribution License (CC BY). The use, distribution or reproduction in other forums is permitted, provided the original author(s) and the copyright owner(s) are credited and that the original publication in this journal is cited, in accordance with accepted academic practice. No use, distribution or reproduction is permitted which does not comply with these terms. 